The BMJ

Cite this as: $B M J 2020 ; 371: m 3978$ http://dx.doi.org/10.1136/bmj.m3978 Published: 19 October 2020

\title{
How do I deal with criticism online?
}

\section{Receiving criticism from another medical professional online can be hurtful or upsetting. Abi Rimmer speaks to three experts about what you can do when this happens}

Abi Rimmer

\section{Discuss with an impartial colleague or friend}

Marina Yiasemidou, National Institute for Health Research academic clinical lecturer in general surgery and specialty trainee year 6 in colorectal surgery, says, "In the era of social media, it's practically impossible to avoid criticism online. On the other hand, having an online presence can be beneficial. It can provide quick access to emerging evidence, allow clinicians to debate, and facilitate the establishment of national and international scientific collaborations.

"I've developed four principles for dealing with being on the receiving end of online criticism. Firstly, never say something online that you would consider too rude to say to someone's face.

"Secondly, always maintain a civil and polite conversation, whether or not the person criticising is willing to do so. Thirdly, reflect whether the criticism is justified and tackle any fair points that have been made. Finally, do not take personally any criticism that isn't constructive.

"I've also developed some practical steps to deal with online criticism. Firstly, I select an impartial member of my team to debate the criticism I received. I ask them if it's justified and what we can we do about improving things.

'I then have a 'cooling off' period before responding and I read my responses twice before I press send. I'll also ask the most diplomatic member of my household or a friend what they think about my response and if they'd word it the same way.

"An important distinction should be between constructive and unjustified comments. In my experience, constructive comments don't include criticism only-as nothing can be 'all bad'-and include a suggestion on how to improve. Comments that are annihilating are rarely justified and have little to teach you."

\section{Take time to think and reflect}

Matt Morgan, consultant in intensive care medicine, says, "You wake up the morning after writing that tweet you were so proud of. Even before breakfast, you check your notifications. One hundred likes, ten comments-life is good. Until you read them.

Colleagues from across the world tell you how wrong you are, point out the cracks in your argument, and you no longer feel like breakfast. This has happened to me many times, it will happen again, and it feels awful.

"The first thing I do is to think and wait. Do they have a point? Reflecting on your words, you may agree with some comments. Although you don't have to respond, I will often say, 'Thanks for pointing that out, good point,' and move on with my day. Reflective practice can happen through social media as much as in real life.

"Not all criticism, however, is positive. Social media can intrude on your breakfast, your day, and your life. You wouldn't expect the postman to shout insults through your letterbox and neither should you acquiesce online. Any offensive comments, I block or report. The mute button can be your friend, allowing you to lock that metaphorical letterbox so the postman can only shout into an empty, echoing void.

"If by lunchtime those tweets are still running around your head, try to remember how fickle and fleeting the virtual world can be. An hour later another fury of fluff will arrive at another person's breakfast table. Try not to add to the noise now you know what it feels like.

"But this is only my take on the matter, feel free to tell me I'm wrong. Just don't shout through my letterbox, I won't be in.”

\section{Avoid retaliation}

Sati Heer-Stavert, Birmingham GP and blogger at unexaminedmedicine.org, says, "In these circumstances, the medium used to send the message is a message itself. Specifics aside, ask yourself why a colleague found it necessary to post criticism on a public forum, online.

"Was it an attempt at well meant, constructive criticism or intentional maliciousness? Was the criticism designed to bait you into an inflammatory response or shame you into action? Alternatively, your colleague may have been trying to damage your reputation while somehow improving their own in a display of medical jousting.

"It's important to remember that in a world of anonymous posters, manufactured controversy, and fake comments, the authenticity of online criticism is often questionable, regardless of the number of likes, follows, or re-tweets the poster has. Online criticism does, however, have the potential to bleed out of the virtual world and into real life where it could affect your wellbeing and those around you.

"It's important, therefore, to avoid validating this process with a retaliatory post. Instead, speak directly to someone you trust, asking for their thoughts. Ask them whether they feel there is any justification for the criticism. You may also want to raise the criticism with your appraiser, educational supervisor, or someone similar. 
"Your organisation may also have procedures in place for managing online infringements of professional behaviour. These options are likely to be much more insightful than fuelling an online flame war." 